\title{
18. A NOTE ON THE HEAD ECHO PROBLEM*
}

\author{
J. RAJCHL \\ (Astronomical Institute of the Czechoslovak Academy of Sciences, Ondřejov)
}

\begin{abstract}
A model of the head echo is examined, in which, as a source of ionization and recombination, an interaction layer of impinging atmospheric molecules $\left(\mathrm{O}_{2}\right)$ mixing with reflected ones is assumed. Dissociative recombination is considered as the most probable process. If we suppose that the interaction layer is that arising in nearly free molecular flow with no shock wave present, we obtain, using the observed heights of head echoes, dimensions of meteoroids in the range $10-1 \mathrm{~cm}$ for absolute magnitudes between -10 and +2 . It is shown that such a layer may be used to explain the high initial recombination coefficient of the order of $10^{-5} \mathrm{~cm}^{3} / \mathrm{sec}$. Using the ionization collision cross-sections measured by Utterback (1963) we obtain for the mean extent of the interaction layer a value $1-100 \mathrm{~m}$ for heights $80-130 \mathrm{~km}$, in accordance with radar observations.
\end{abstract}

A successful explanation of the origin of the meteor head echo is at present far from definitive. The fundamental problems are:

(1) the mechanism of ionization before intense evaporation is present, including the main ionized constituent responsible for the head echo;

(2) the extent of this zone of ionization, of the order of tens or hundreds of metres;

(3) the quick de-ionization within this zone.

Principally, two mechanisms of ionization may be suggested: (1) the UV radiation generated by the meteor, and (2) the atmospheric particles reflected from the meteor body. The UV hypothesis proposed by McKinley and Millman (1949) was developed by Cook and Hawkins (1960) quantitatively but without a detailed solution of the process of UV-radiation generation.

In this note a model is presented where, as a source of ionization and recombination, a zone of interacting particles is supposed. The particles are the atmospheric $\mathrm{O}_{2}$ molecules interacting with those $\mathrm{O}_{2}$ reflected from the meteor body. As has been calculated by Rajchl (1964), this mechanism gives about $\geqslant 10^{13}$ electrons/cm for meteoroids of $1-10 \mathrm{~cm}$ in size.

The coefficient of de-ionization, as deduced by Cook and Hawkins (1960) and by McIntosh (1962) is extraordinarily high, of the order of $10^{-5} \mathrm{~cm}^{3} / \mathrm{sec}$. Thus we can adopt as possible only processes with highest known recombination coefficient (i.e. about $10^{-6}-10^{-7} \mathrm{~cm}^{3} / \mathrm{sec}$ ); these are: (1) dissociative recombination, (2) mutual neutralization for molecular and three-body recombination, $e+e+A^{+} \rightarrow A^{\prime}+e$

* Details of this paper will be published in Bull. astr. Inst. Csl.

Kresák and Millman (eds.), Physics and Dynamics of Meteors, 187-189. (C) I.A.U. 
(Robinson, 1965), for atomic ionized species. As mutual neutralization is ineffective in the removal of free electrons and three-body recombination is quickly decreasing with temperature, we adopt as most probable the dissociative recombination of molecular ions. To overcome the difference of about two orders between the known and required values of the recombination coefficient, we consider a concept of initial recombination (the coefficient $\alpha$ ) instead of the equilibrium volume recombination (the coefficient $\alpha_{\infty}$ ), as formulated by Ebert (1964). We obtain that for a model of an interaction layer of thickness of $0 \cdot 1-1 \mathrm{~cm}$ and electron densities higher than $10^{11} \mathrm{el} / \mathrm{cm}$ the recombination is more efficient than diffusion, and the recombination coefficient given by

$$
\alpha=\alpha_{\infty} \underset{\bar{n}(0)\left(8 \pi D t+\pi r_{0}^{2}\right)}{\bar{N}(0)}=\frac{\overline{N(0)}}{n(0) \pi r_{0}^{2}},
$$

where $\overline{N(0)}$ and $\overline{n(0)}$ are the average linear and volume electron densities respectively, for the time $t=0 . D$ is the diffusion coefficient; $r_{0}$, the interaction-layer thickness, is of the order of $10^{-5} \mathrm{~cm}^{3} / \mathrm{sec}^{*}$

From the gas-dynamic point of view, such interaction layer may be present only in the nearly free molecular flow or in the transition and slip flow. However, at lower heights, i.e. in the transition and slip-flow conditions, a shock front is formed and the reflected-molecules mechanism is hardly possible. Therefore, we suppose that the head echo process is present in nearly free molecular flow conditions. Using the heights of head echoes published by Millman and McKinley (1963) we deduce, for nearly free molecular conditions $(B=1-0 \cdot 1)$, the meteoroid dimensions in the interval $10-1 \mathrm{~cm}$, for magnitudes from $-10^{\mathrm{m}}$ to $+2^{\mathrm{m}}$.

Using the collision cross sections for ionization of $\mathrm{O}_{2}$ by $\mathrm{O}_{2}, Q_{\mathrm{i}} \approx 10^{-15}-10^{-16} \mathrm{~cm}^{2}$ (Utterback, 1963), we obtain, for the extent of the interaction layer (instead of the gas-kinetic mean free path), from the equation

$$
\lambda_{\mathrm{i}}=\begin{gathered}
1 \\
n Q_{\mathrm{i}}
\end{gathered}
$$

( $n=$ the number density of $\mathrm{O}_{2}$ in $\mathrm{cm}^{-3}$ ) a value of $100-1 \mathrm{~m}$ within the height interval $130-80 \mathrm{~km}$, i.e. consistent with dimensions obtained from radar observations (McIntosh, 1963) for medium- and high-velocity meteors. Comparing the cross sections for excitation and diffusion with those for ionization, we find that the extent of 'diffusion' and 'excitation' zones is about one order higher, except for the low-velocity meteors, where the diffusion layer is nearer to the meteoroid than the ionization layer.

* Another possibility of higher recombination coefficient may consist in the presence of $\mathrm{O}_{3}{ }^{+}$ molecules instead of $\mathrm{O}_{2}^{+}$(Biondi, 1964). These complex molecules are formed by pressures higher than $10^{-2}$ torr (dynamic pressure produced by meteors is higher than 2 torr) with recombination coefficient $\geqslant 10^{-6} \mathrm{~cm}^{3} / \mathrm{sec}$. But this value is now confirmed only for $\mathrm{N}_{4}^{+}$, not for $\mathrm{O}_{3}{ }^{+}$! 
In conclusion, we point out that:

(1) from our point of view a shock front seems not to be a source of the head echo; on the contrary, it works against the head-echo development (the distance of shock front is $10^{-1:-2}$ of body diameter).

(2) with respect to the definitive determination of the ionized constituent, the correlation between the head echo and the occurrence of the forbidden oxygen green line in meteors pointed out anew recently by the present writer (Rajchl, 1965) may be very useful. If this correlation is real, then, from the viewpoint of the greenline origin, dissociative recombination of $\mathrm{NO}^{+}$and $\mathrm{N}_{2}^{+}$would be excluded as sources for head echo origin and conversely, from the point of view of head echo origin, mutual neutralization and the well-known Chapman's reaction must be excluded as possible sources of the green-line occurrence in meteor spectra. Thus, it is seen to be desirable to clear up the question about the reality of this correlation.

\section{References}

Biondi, M.A. (1964) Ann. Géophys., 20, 34.

Cook, A.F., Hawkins, G.S. (1960) Smithson. Contr. Astrophys., 5, 1.

Ebert, H.G. (1964) Z. Phys., 181, 181.

McIntosh, B. A. (1962) J. atmos. terr. Phys., 24, 311.

McIntosh, B. A. (1963) Can. J. Phys., 41, 355.

McKinley, D. W. R., Millman, P. M. (1949) Proc. IRE, 37, 364.

Millman, P. M., McKinley, D.W.R. (1963) in The Moon, Meteorites and Comets, ed. by B. M. Middlehurst and G.P. Kuiper, University of Chicago Press, p. 674.

Rajchl, J. (1964) Bull. astr. Inst. Csl., 15, 137.

Rajchl, J. (1965) Bull. astr. Inst. Csl., 16, 282.

Robinson, B. B. (1965) J. geophys. Res., 70, 3793.

Utterback, N.G. (1963) Phys. Rev., 129, 219. 